\title{
Repetitive occurrence of explosive events at a coronal hole boundary
}

\author{
J. G. Doyle ${ }^{1}$, M. D. Popescu ${ }^{1,2}$, and Y. Taroyan ${ }^{1}$
}

\author{
1 Armagh Observatory, College Hill, Armagh BT61 9DG, N. Ireland \\ e-mail: jgd@arm.ac.uk \\ 2 Astronomical Institute of the Romanian Academy, RO-040557 Bucharest 28, Romania
}

Received 14 July 2005 / Accepted 20 September 2005

\section{ABSTRACT}

SUMER/SoHO data taken at a coronal hole boundary show a repetitive explosive event occurrence rate of around 3 min increasing to over 5 min towards the end of the activity. We suggest that the neighbouring oppositely directed closed and open field lines at the coronal hole boundary undergo repetitive reconnection seen as a sequence of explosive events. The repetitive reconnection may be triggered by transverse oscillations of the flux tubes in the closed field line region. These oscillations periodically separate and bring together the closed and open field lines on the two sides of the coronal hole boundary. An important indicator favouring the interpretation in terms of a kink mode is the observed increase in the oscillation period.

Key words. Sun: activity: - Sun: oscillations - Sun: transition region - Sun: UV radiation - line: profiles

\section{Introduction}

Explosive events (also called bidirectional jets) are small regions of a few arcseconds size with strong blue- and red-shifted emission reaching Doppler shifts of up to $150 \mathrm{~km} \mathrm{~s}^{-1}$. Over the past decade, there have been many studies of explosive event (EE) activity. For example, Chae et al. (1998) found that EEs appear preferably in regions with weak fluxes of mixed polarity or on the border of regions with large concentration of magnetic flux. Madjarska \& Doyle (2002) investigated time delays between the chromospheric and transition region lines. Teriaca et al. (2004) estimated that the average size of an EE was $1800 \mathrm{~km}$ with a birthrate of $2500 \mathrm{~s}^{-1}$ over the entire Sun based on the O VI $1032 \AA$ line. The jets' number density along coronal hole $(\mathrm{CH})$ boundaries was found by Madjarska et al. (2004) to be about 4-5 times higher with respect to the quiet Sun, while Doyle et al. (2005) reported on EEs which were clearly visible in N v $1238 \AA$ yet were very weak or absent in O v $629 \AA$.

Dere (1994) was the first to note that EEs are often observed in bursts lasting up to $30 \mathrm{~min}$ in regions undergoing magnetic cancellation. This was confirmed by Pérez et al. (1997), Chae et al. (1998) and Ning et al. (2004). It is this point that the present study is directed towards. Here, we look at time series data taken with SUMER (Solar Ultraviolet Measurements of Emitted Radiation) onboard SoHO (Solar \& Heliospheric Observatory) in a polar $\mathrm{CH}$ region over a period of more than

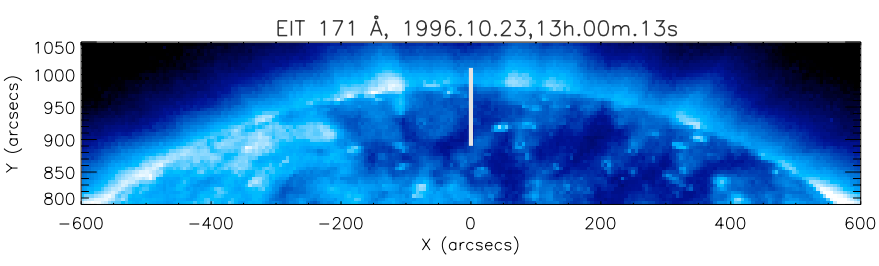

Fig. 1. The coronal hole region from the Sun's north pole in the light emitted by Fe IX/X 171 A observed with EIT on 23 October 1996. The white line shows the fixed position of the SUMER slit. The image was taken 21 min after the beginning of the SUMER time series.

$4 \mathrm{~h}$ and in particular, we search for evidence of whether these bursts are random or have a periodical nature.

\section{Data}

The data presented here were acquired with the SUMER spectrometer (Wilhelm et al. 1995; Lemaire et al. 1997) in the north polar $\mathrm{CH}$ on 23 October 1996. Slit no. 8 (0.3" wide and $120^{\prime \prime}$ long) with detector B was fixed at $x=0^{\prime \prime} ; y=$ $950.25^{\prime \prime}$. Figure 1 shows the position of the slit on an EIT (Extreme ultraviolet Imaging Telescope) image taken during our time series. The SUMER dataset was taken between 12:39:01-16:51:19UT, with an exposure time of $\approx 10.5 \mathrm{~s}$. SUMER data has a spatial resolution of $1^{\prime \prime}$ (i.e. the pixel size along the slit).

The line in which the observation was transmitted to Earth is $\mathrm{O}$ VI $1032 \AA$ (originating at $\approx 300000 \mathrm{~K}$ in the transition 


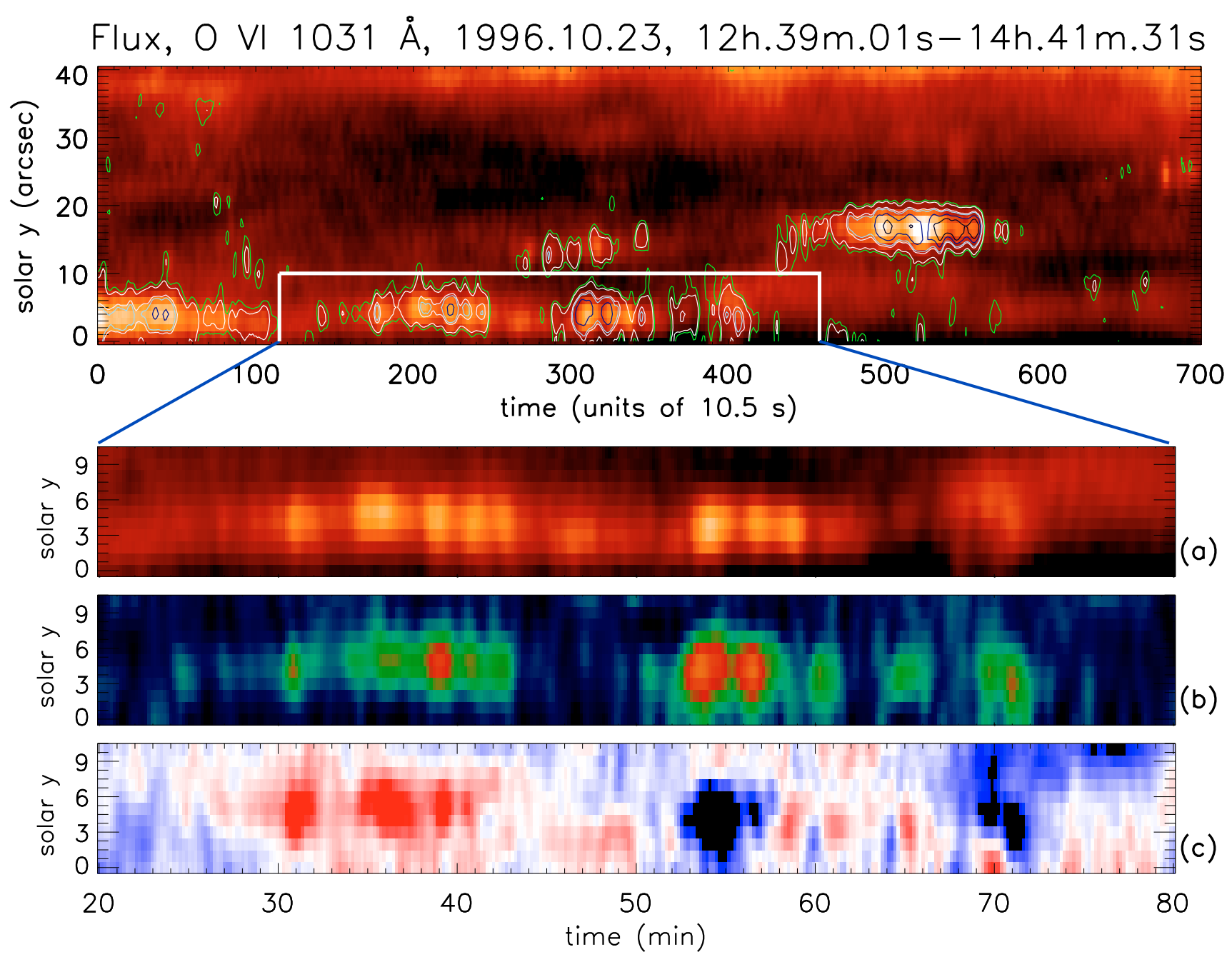

Fig. 2. Top: a sequence of $\approx 122 \min \left(700\right.$ exposures of $10.5 \mathrm{~s}$ each) and $40^{\prime \prime}$ on solar- $y$ from the time series flux map of O VI $1032 \AA$ observed with SUMER on 23 October 1996 (logarithmic scale). Over-plotted are the contours of the line widths. The white rectangle represents the selected burst of EEs (60 min; 10") shown in more detail on the bottom panels: a) the flux map (logarithmic scale); b) line widths (logarithmic scale); c) the Doppler velocity (linear scale; $\pm 20 \mathrm{~km} \mathrm{~s}^{-1}$ ).

region). This line shows a very good response to EEs (Teriaca et al. 2004). We applied the standard procedures for correcting and calibrating the raw data: decompression, reversal, flatfield, dead-time, local-gain and geometrical correction (e.g. see Xia et al. 2005 for further details or the SUMER/SoHO homepage).

\section{Results}

In this contribution, we only focus on the location with the biggest concentration of EEs, e.g. towards the base of the slit. The upper panel in Fig. 2 shows the O VI $1032 \AA$ flux for a $40^{\prime \prime}$ section over $\approx 122 \mathrm{~min}$ (700 exposures of $10.5 \mathrm{~s}$ each). Over-plotted are the contours of the full width at half maximum $(F W H M)$ which give an indication of where EEs take place. It is obvious that the largest EE activity is at the base of the time series map. If we check the EIT image, we see that the base of the slit is located right on the intersection between the base of the dark $\mathrm{CH}$ and the top of a bright feature (a $\mathrm{CH}$ boundary). We consider solar- $y=0$ as the base of our slit $\left(y=890.25^{\prime \prime}\right.$ in solar coordinates, $\approx 70^{\prime \prime}$ down from the solar limb).
We further select only one example where a sequence of EE repetition is seen (the white rectangle, 10" high; $60 \mathrm{~min}$ time length) which is shown in more detail in the bottom panels of Fig. 2. In (a) we plot the O VI integrated flux, which is done by summing all 50 spectral pixels of the SUMER window and subtracting the continuum background. In (b) we show the $F W H M$, and in (c) the Doppler velocity. These two parameters were computed by fitting the line with a single Gaussian profile. Although this is a good approximation for the "quiet" transition region, a single profile is not really what we see when an EE occurs. The Doppler map is hence only an indication of excess blue/red wings in the line. As noted by Teriaca et al. (2004), line profiles which show at least one of the fitted parameters (or the $\chi^{2}$ ) diverging by more than $3 \sigma$ from the average of its distribution are potential EEs.

During the whole observation ( $\approx 4 \mathrm{~h} 12 \mathrm{~min}$ ), the base of the slit moved $\approx 9^{\prime \prime}$ on the solar disk (the rotational compensation was switched off, as we are very close to the limb). Inspecting the EIT image, we see that only in the initial stage was the slit located on a $\mathrm{CH}$ boundary; as we progress in time, the slit 
(a)
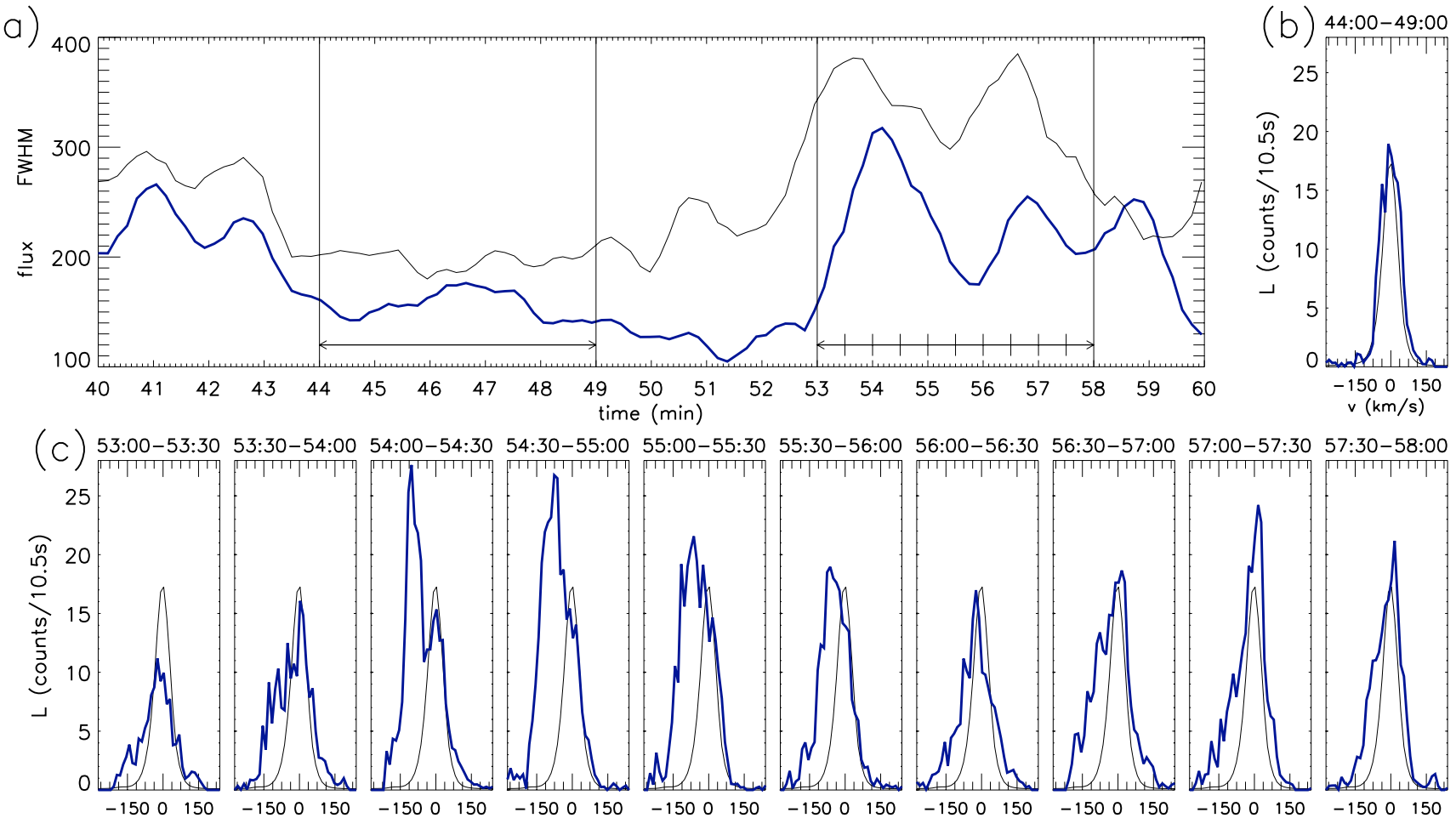

Fig. 3. Panel a) is a sequence of $20 \mathrm{~min}$ for the flux (in counts/10.5 s, the thin line) and the $F W H M$ (in arbitrary units, the thick line) variation (averaged over 3" at solar- $y=3-5$ ). Panel b) is the spectral radiance, $L$, integrated over 5 min in a "quiet" region (position 44 to 49 in panel a). Panel $\mathbf{c}$ ) is the spectral radiance integrated over $\approx 30 \mathrm{~s}$ (an average of 3 exposures of $10.5 \mathrm{~s}$ each) over an interval of 5 min (position 53 to 58 in panel a), showing how rapidly it changes during the EE activity. In panels b) and c), the average spectral radiance in a quiet region from the $\mathrm{CH}$ is plotted as a thin line.

enters a "dark" region in the $\mathrm{CH}$. This is why towards the second part of the observation the high EE activity from the base of the slit vanishes. During the $\approx 60$ min interval of our selected EE sequence, the base of the slit moved $\approx 2.1^{\prime \prime}$. From Fig. 2 it is obvious that these EEs undergo a repetitive sequence. In order to estimate what is the repetition rate of the EEs occurrence, we applied the wavelet transform to the $F W H M$ variation with time at solar- $y=3-5$ (see Fig. 2). Details on the wavelet analysis were originally given by Torrence \& Compo (1998) ${ }^{1}$. We basically apply the transform as described in Popescu et al. (2005). For the convolution of the time series, we chose the Morlet function, and to establish if the oscillations found are real, we implemented the Linnell Nemec \& Nemec (1985) randomization method, which estimates the significance level of the peaks in the wavelet spectrum. The use of the randomization technique was done according to O'Shea et al. (2001). In Fig. 3 we show some examples of the spectral profile of these EEs which clearly have a secondary blue-shifted component, sometimes with little or no increase in the intensity.

The top panel of Fig. 4 shows the time variation of the FWHM in spectral pixels averaged over 3 " on solar-y. The left bottom panel is the wavelet spectrum; and the right panel is the global wavelet spectrum, which is the sum of the wavelet power over time at each oscillation period. The dark-coloured regions from the wavelet spectrum show the locations of the highest power. Cross-hatched regions indicate the "cone of influence",

\footnotetext{
${ }^{1}$ http://paos.colorado.edu/research/wavelets/
}

where edge effects become important. Only periods less than 11 min are considered.

For the series of EEs starting around $t=50 \mathrm{~min}$, the wavelet analysis detects two classes of periods. A weak, less significant "constant" period of around 8 min plus a stronger signal which increases in period with time. The repetitive rate is initially around $3 \mathrm{~min}$ increasing to over $5 \mathrm{~min}$ towards the end of the activity. We clearly see six cycles in an interval of $\approx 25 \mathrm{~min}$ (from $t=50$ up to $75 \mathrm{~min}$ ). The weaker "constant" period is present throughout the observations and is not related to the increasing period.

\section{Discussion}

As outlined in the Introduction, several previous investigations have indicated that EEs sometimes tend to occur in bursts. For example, Ning et al. (2004) found that events can occur 3-5 min apart and suggested that oscillations due to some wave motion could play a role in triggering the individual events. Fan et al. (2004) adopted a 2D MHD approach involving anomalous resistivity. Although such an approach can produce a burst-like appearance, it is difficult to see how it could produce the periodlike events seen in Fig. 4.

$\mathrm{CH}$ boundaries are regions where the magnetic field changes its configuration. In particular, oppositely directed closed and open field lines may co-exist at the boundary. Therefore, favorable conditions for the occurrence of magnetic reconnection are present. The reconnection between the 

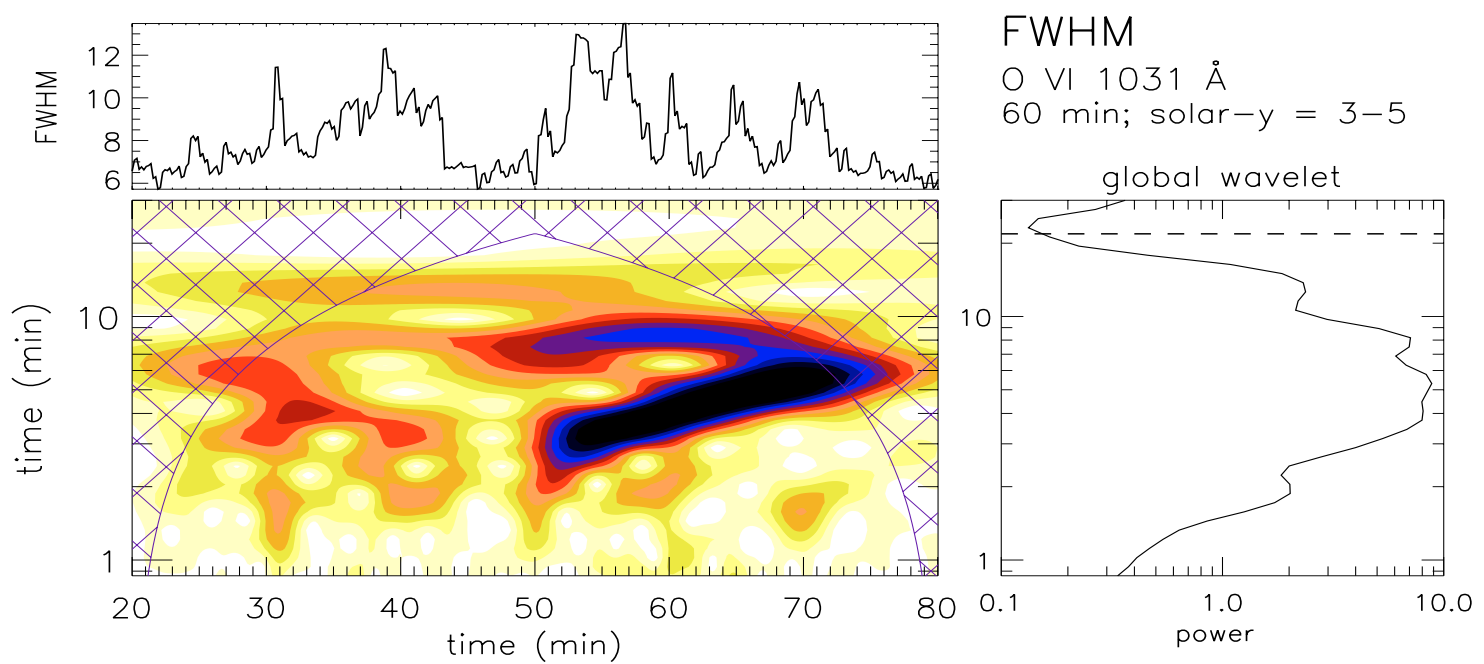

Fig. 4. Wavelet results for the time variation of O VI $1032 \AA$ over $60 \mathrm{~min}$, at solar- $y=3-5$ (averaged over $3^{\prime \prime}$ ). The top panel shows the variation of the FWHM in spectral pixels. The corresponding wavelet power spectrum is shown in the bottom middle panel. In the global wavelet power spectra the top dashed line represents the maximum allowed period ( $\approx 29 \mathrm{~min})$. The other lines represent the periods that appear in the selected example of $F W H M$ variation with time.

neighbouring and oppositely directed field lines is likely to be observed as an EE or a bidirectional jet, as shown by the increased rate of EE activity at a $\mathrm{CH}$ boundary by Madjarska et al. (2004). The repetitive character of these events and the 3-5 min periodicities seen in Fig. 4 imply that MHD waves could be involved in this process. Recent SoHO/TRACE observations have revealed the presence of a great variety of MHD waves in the solar atmosphere. Observational and theoretical aspects of MHD waves in the solar atmosphere are discussed in review papers by Aschwanden (2003) and Roberts (2004). In the following discussion, we assess the likelihood of different types of waves to trigger EEs and modulate their occurrence rate.

Three different classes of MHD waves may exist: Alfvén waves and slow/fast magnetoacoustic waves. The solar atmosphere essentially represents a low- $\beta$ plasma environment where the magnetic pressure dominates the plasma pressure. Therefore, the slow waves are essentially acoustic waves constrained to propagate along the strong magnetic field lines. These waves have negligible effect on the strength and orientation of the magnetic field and are unlikely to play an important role in the above described reconnection process. Alfvén waves are nevertheless believed to be relevant to many processes occurring in the solar atmosphere. These waves twist the field lines along which they propagate. In the present situation, however, it is unclear how the twisting could create more favorable conditions for the reconnection between the neighbouring closed and open oppositely directed field lines. We therefore examine the possibility of a mechanism involving fast waves.

The $\mathrm{CH}$ boundary region separates the tenuous $\mathrm{CH}$ region from the denser quiet sun region. The equation of magnetohydrostatic pressure balance requires the total pressure (plasma and magnetic) to remain constant across the boundary:

$p+\frac{B^{2}}{2 \mu}=$ const.
Equation (1) can be rewritten in the form

$\rho\left(\frac{c_{\mathrm{s}}^{2}}{\gamma}+\frac{c_{\mathrm{A}}^{2}}{2}\right)=$ const.

where $\rho$ is the density and

$c_{\mathrm{s}}=\left(\frac{\gamma p}{\rho}\right)^{\frac{1}{2}}, \quad c_{\mathrm{A}}=\left(\frac{B^{2}}{\mu \rho}\right)^{\frac{1}{2}}$

are the sound and Alfvén speeds. Equation (2) implies that the Alfvén speed in the $\mathrm{CH}$ region exceeds the Alfvén speed in the neighbouring quiet sun region provided they have the same temperature. The fast waves are ducted by the inhomogeneity in the Alfvén speed and may propagate in the region where the Alfvén speed is lower (Edwin \& Roberts 1983). The field lines in this region are closed. The observations presented in this paper were done in the $\mathrm{O}$ VI line which has a peak formation temperature of about $300000 \mathrm{~K}$. Assuming that the electron temperature is approximately the same as the ion temperature, we find a sound speed of about $\sim 85 \mathrm{~km} \mathrm{~s}^{-1}$. This gives a lower estimate for the phase speed of the fast waves (the phase speed of the fast waves exceeds the minimum Alfvén speed which, in turn, exceeds the sound speed). The observed period $P$ in Fig. 4 is in the range of 3-5 min. Taking a typical flux tube width of $d=1 \mathrm{Mm}$ and assuming that $c_{\mathrm{A}} \approx 2 c_{\mathrm{s}}$, we find that the corresponding dimensionless wavenumber is small:

$k d<\frac{2 \pi d}{c_{\mathrm{A}} P} \approx 0.2$,

and, therefore, the thin flux tube approximation can be applied. Three different types of waves can be generated in thin flux tubes: slow (longitudinal), Alfvén (torsional) and kink (transverse) waves. These waves are governed by the Klein-Gordon equation and have their characteristic cut-off frequencies (Roberts 2004). As we eliminated the first two types of waves, we discuss the kink mode. 
The kink mode oscillations shake the field lines of the flux tube in the transverse direction. Such transverse motions could periodically separate and bring together the closed and open field lines at the boundary and thus trigger magnetic reconnection between oppositely directed field lines. Another important indicator favouring the interpretation of the repetitive EEs in terms of a kink mode is the period increase seen in Fig. 4. The fact that the tube oscillates does not contradict the requirement of hydrostatic pressure balance as the amplitude of the oscillations decreases exponentially outside the tube. The thinner the tube, the smaller the wave amplitude away from the tube (Edwin \& Roberts 1983).

It is well-known that EEs are associated with the cancellation of the magnetic flux. On the other hand, the frequency of the kink mode is proportional to the Alfvén frequency. The Alfvén speed would gradually decrease due to the flux cancellation and this would result in an increase in the period of the kink mode, i.e. as the outer field lines of the flux tube get reconnected, they are substituted by the inner field lines. This is a continuous process during which the Alfvén speed gradually decreases because the outer field lines "wear off", i.e., the strength of the magnetic field decreases.

Standing and propagating kink mode oscillations are readily observed in the higher regions of the solar atmosphere. Standing kink mode oscillations could be excited impulsively. They have damping times of about 3 wave periods (e.g. Aschwanden et al. 1999). The kink modes could also be excited at photospheric heights due to the interaction between the flux tubes embedded in the convection zone and the external motions (see, e.g., Musielak \& Umlschneider 2001; De Pontieu et al. 2004). Provided the series of EEs seen in Fig. 2 (from $t=$ $50 \mathrm{~min}$ to $75 \mathrm{~min}$ ) were consecutively triggered by kink mode oscillations, the damping time of these oscillations should be long (about 5-10 wave periods). A possible candidate for the damping of standing kink modes is the mechanism of resonant absorption (Ruderman \& Roberts 2002). The damping time is given by the expression

$\tau=\frac{d}{\pi l} \frac{\rho_{0}+\rho_{\mathrm{e}}}{\rho_{0}+\rho_{\mathrm{e}}}$

where $\rho_{0}$ is the density inside the tube and $\rho_{\mathrm{e}}$ is the density outside the tube, the transition being accomplished over a distance $l \ll d$ to the boundary of the tube. Equation (4) shows that the thinner the transitional layer where the Alfvén speed decreases, the longer the damping time. In the present case, this layer could become thin due to the continuous interaction of the flux tube with the external magnetic field. An alternative explanation for the observed long damping time is the presence of a continuous/intermittent driver.

\section{Conclusions}

In summary, the following picture has emerged. The neighbouring oppositely directed closed and open field lines at the $\mathrm{CH}$ boundary undergo repetitive reconnection seen as explosive events. The repetitive reconnection is triggered by transverse oscillations (i.e. the kink mode) of the flux tubes in the closed field line region. These oscillations periodically separate and bring together the closed and open field lines on the two sides of the coronal hole boundary. This picture is somewhat similar to what is seen in the magnetosphere: a sudden increase in the solar wind pressure during southward interplanetary magnetic field orientation leads to enhanced magnetic reconnection on the dayside magnetopause (Boudouridis et al. 2005). Hence, the proposed mechanism explains the repetitive nature of the observed EEs, however, other possibilities should not be excluded.

Acknowledgements. Armagh Observatory's research is grant-aided by the N. Ireland Dept. of Culture, Arts \& Leisure. This work was partially supported by the Program for Research in Irish Third Level Institutions for Grid-enabled Computational Physics of Natural Phenomena (Cosmogrid) \& by PPARC grant PPA/G/S/2002/00020. SUMER is financially supported by DLR, CNES, NASA \& ESA PRODEX programme (Swiss contribution). SUMER \& EIT are part of SoHO which is a mission of international cooperation between ESA and NASA. We thank the referee for valuable comments.

\section{References}

Aschwanden, M. 2003, Review of coronal oscillations, in Turbulence, Waves and Instabilities in the Solar Plasma, ed. Erdélyi et al., NATO Sci. Ser., 124, 215

Aschwanden, M. J., Fletcher, L., Schrijver, C. J., \& Alexander, D. 1999, ApJ, 520, 880

Boudouridis, A., Zesta, E., Lyons, L. R., et al. 2005, J. Geophys. Res. 110, A05214

Chae, J., Wang, H., Lee, C.-Y., Goode, P. R., \& Schühle, U. 1998, ApJ, 497, L109

De Pontieu B., Erdélyi, R., \& James, S. P. 2004, Nature, 430, 536

Dere, K. P. 1994, Adv. Space Res. 14, 13

Doyle, J. G., Ishak, B., Ugarte-Urra, I., Bryans, P., \& Summers, H. P. 2005, A\&A, 439, 1183

Edwin, P. M., \& Roberts, B. 1983, Sol. Phys., 88, 179

Fan, Q., Feng, X., \& Xiang, C. 2004, Communications in Theoretical Physics, 41(5), 790

Lemaire, P., Wilhelm, K., Curdt, W., et al. 1997, Sol. Phys., 170, 105

Linnell Nemec, A. F., \& Nemex, J. M. 1985, AJ, 90, 2317

Madjarska, M. S., \& Doyle, J. G. 2002, A\&A, 382, 319

Madjarska, M. S., Doyle, J. G., \& van Driel-Gesztelyi, L. 2004, ApJ, 603, L57

Musielak, Z. E., \& Ulmschneider, P. 2001, A\&A, 370, 541

Ning, Z., Innes, D. E., \& Solanki, S. K. 2004, A\&A, 419, 1141

Pérez, M. E., Doyle, J. G., Erdélyi, R., \& Sarro, L. M. 1997, A\&A, 342,279

O’Shea, E., Banerjee, D., Doyle, J. G., Fleck, B., \& Murtagh, F. 2001, A\&A, 368, 1095

Popescu, M. D., Banerjee, D., O'Shea, E., Doyle, J. G., \& Xia, L. D. 2005, A\&A, 442, 1087

Roberts, B. 2004, MHD Waves in the Solar Atmosphere, ed. R. Erdélyi, J. L. Ballester, \& B. Fleck, ESA-SP, 547, 1

Ruderman, M. S., \& Roberts, B. 2002, ApJ, 577, 475

Teriaca, L., Banerjee, D., Falchi, A., Doyle, J. G., \& Madjarska, M. S. 2004, A\&A, 427, 1065

Torrence, C., \& Compo, G. P. 1998, Bull. Am. Meteorological Soc., 79,61

Wilhelm, K., Curdt, W., Marsch, E., et al. 1995, Sol. Phys., 162, 189

Xia, L. D., Popescu, M. D., Doyle, J. G., \& Giannikakis, J. 2005, A\&A, 438, 1115 